International Journal of Pure and Applied Mathematics

Volume 96 No. $4 \quad 2014,427-444$

ISSN: 1311-8080 (printed version); ISSN: 1314-3395 (on-line version)

url: http://www.ijpam.eu

doi: http://dx.doi.org/10.12732/ijpam.v96i4.2

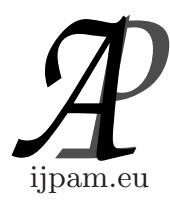

\title{
SOME COMMON FIXED POINT THEOREMS FOR THREE PAIRS OF MAPPINGS VIA WEAKLY COMMUTING AND WEAKLY COMPATIBILITY IN $G$-METRIC SPACES
}

\author{
Surjeet Singh Tomar ${ }^{1}$, Deepak Singh ${ }^{2}$, P.C. Dixit ${ }^{3}$, M.S. Rathore ${ }^{4}$ \\ ${ }^{1}$ Jai Narayan College of Technology \\ Bhopal, M.P., INDIA \\ ${ }^{2}$ Department of Applied Sciences \\ NITTTR, Under Ministry of HRD \\ Govt. of India, Bhopal, (M.P.), 462002, INDIA \\ ${ }^{3}$ Department of Mathematics \\ T.I.T. College, Bhopal, INDIA \\ ${ }^{4}$ Department of Mathematics \\ Chandrashekhar Azad Govt. P.G. College \\ Sehore, M.P., INDIA
}

\begin{abstract}
The purpose of this paper is to establish the existance and uniqueness of fixed point theorems for three pairs of self mappings in the frame work of complete $G$-metric spaces. Our results are obtained by using the contractive conditions weakly commuting for one pair and weakly compatibility for other two pairs. These results generalize and partially extend some recent results in existing literature.
\end{abstract}

AMS Subject Classification: 47H10, 54H 25

Key Words: complete $G$-metric space, weakly commuting mappings, weakly compatible mappings, contractive conditions

Received: November 10, 2013

(C) 2014 Academic Publications, Ltd. url: www.acadpubl.eu

${ }^{\S}$ Correspondence author 


\section{Introduction}

The concept of $D$-metric spaces was introduced by Dhage[1],[2],[3],[4]. et al. as generalization of ordinary metric functions and went on to present several fixed point results for single and multivalued mappings. Mustafa and Sims[12] and Naidu et al. [8],[9],[10] demonstrated that most of the claims concerning the fundamental topological structure of $D$ - metric space are incorrect, alternatively, Mustafa and Sims introduced in [12] more appropriate notion of generalized metric space which they called $G$ - metric spaces, and obtained some topological properties. Later Zead Mustafa, Hamed Obiedat and Fadi Awawdeh[13], Mustafa, Shatanawi and Bataineh [14], Mustafa and Sims [15] Shatanawi [11] and Renu Chugh, Tamanna Kadian, Anju Rani and B.E. Rhoades[7] et al. obtained some fixed point theorems for a single map in $G$ - metric spaces.

The purpose of this paper is to use the concept of weakly commuting mappings and weakly compatible mappings to discuss some new common fixed point problems for six self-mappings in $G$-metric spaces. First, we present some known definitions and propositions in $G$ - metric spaces .

\section{Preliminaries}

Now we give basic definitions and some basic results which are helpful for proving our main result.

In 2006, Mustafa and Sims [12] introduced the concept of G-metric spaces as follows:

Definition 2.1. [12] Let $X$ be a nonempty set, and let $G: X \times X \times X \rightarrow$ $R^{+}$be a function satisfying the following properties:

1. $(G-1) G(x, y, z)=0$ if $x=y=z$;

2. (G-2) $0<G(x, x, y)$, for all $x, y \in X$ with $x \neq y$.

3. (G-3) $G(x, x, y) \leq G(x, y, z)$ for all $x, y, z \in X$ with $y \neq z$

4. $(G-4) G(x, y, z)=G(x, z, y)=G(y, z, x)=\ldots \ldots$. symmetry in all three variables,

5. (G-5) $G(x, y, z) \leq G(x, a, a)+G(a, y, z)$ for all $x, y, z, a \in X$

The function $G$ is called a generalized metric or a $G$-metric on $X$ and the pair $(X, G)$ is called a $G$-metric space. 
Definition 2.2. [12] Let $(X, G)$ be a $G$-metric space, and let $\left\{x_{n}\right\}$ be a sequence of points of $X$, a point $x \in X$ is said to be the limit of the sequence $\left\{x_{n}\right\}$, if $\lim _{n, m \rightarrow \infty} G\left(x, x_{n}, x_{m}\right)=0$ and we say that the sequence $\left\{x_{n}\right\}$ is $G$ convergent to $x$ or $\left\{x_{n}\right\} G$-convergent to $x$.

Thus, $x_{n} \rightarrow x$ in a $G$-metric space $(X, G)$ if for any $\epsilon>0$, there exists $k \in N$ such that $G\left(x, x_{n}, x_{m}\right)<\epsilon$ for all $m, n \geq k$.

Proposition 2.1. [12] Let $(X, G)$ be a $G$-metric space. Then the following are equivalent:

1. $\left\{x_{n}\right\}$ is $G$-convergent to $x$;

2. $G\left(x_{n}, x_{n}, x\right) \rightarrow 0$ as $n \rightarrow \infty$;

3. $G\left(x_{n}, x, x\right) \rightarrow 0$ as $n \rightarrow \infty$;

4. $G\left(x_{n}, x_{m}, x\right) \rightarrow 0$ as $n, m \rightarrow \infty$;

Definition 2.3. [12] Let $(X, G)$ be a $G$-metric space, a sequence $\left\{x_{n}\right\}$ is called $G$-Cauchy if for every $\epsilon>0$, there is $k \in N$ such that $G\left(x_{n}, x_{m}, x_{l}\right)<\epsilon$ for all $n, m, l \geq k$; that is $G\left(x_{n}, x_{m}, x_{l}\right) \rightarrow 0$ as $n, m, l \rightarrow \infty$.

Proposition 2.2. [12] Let $(X, G)$ be a $G$-metric space. Then the following are equivalent:

1. The sequence $\left\{x_{n}\right\}$ is $G$-cauchy;

2. For every $\epsilon>0$, there is $k \in N, G\left(x_{n}, x_{m}, x_{m}\right)<\epsilon$ for all $n, m \geq k$;

Definition 2.4. $[12]$ Let $(X, G)$ and $\left(X^{\prime}, G^{\prime}\right)$ be $G$-metric space and let $f:(X, G) \rightarrow\left(X^{\prime}, G^{\prime}\right)$ be a function. Then $f$ is said to be $G$-continuous at a point $a \in X$ if and only if, for every $\epsilon>0$, there is $\delta>0$ such that $x, y \in X$ and $G(a, x, y)<\delta$ imply $G^{\prime}(f(a), f(x), f(y))<\epsilon$. A function $f$ is $G$-continuous at $X$ if and only if it is $G$-continuous at $a \in X$.

Proposition 2.3. [12] Let $(X, G)$ be a $G$-metric space. Then the function $G(x, y, z)$ is jointly continuous in all three of its variables.

Definition 2.5. [12] A $G$-metric space $(X, G)$ is said to be $G$-complete if every $G$-Cauchy sequence in $(X, G)$ is $G$-convergent in $X$.

Definition 2.6. [5] Two self mappings $f$ and $g$ of a $G$-metric space $(X, G)$ are said to be weakly commuting if $G(f g x, g f x, g f x) \leq G(f x, g x, g x)$ for all $x \in X$. 
Definition 2.7. [5] Let $f$ and $g$ be two mappings from a $G$-metric space $(X, G)$ into itself. Then the mappings $f$ and $g$ are said to be weakly compatible if $G(f g x, g f x, g f x)=0$ whenever $G(f x, g x, g x)=0$.

Proposition 2.4. [12] Let $(X, G)$ be a $G$-metric space. Then for all $x, y, z, a \in X$, it follows that:

1. If $G(x, x, y)=0$ then $x=y=z$;

2. $G(x, y, z) \leq G(x, x, y)+G(x, x, z)$;

3. $G(x, y, y) \leq 2 G(y, x, x)$;

4. $G(x, y, z) \leq G(x, a, z)+G(a, y, z)$;

5. $G(x, y, z) \leq \frac{2}{3}(G(x, y, a)+G(x, a, z)+G(a, y, z))$;

6. $G(x, y, z) \leq G(x, a, a)+G(y, a, a)+G(z, a, a)$;

\section{Main Results}

Theorem 3.1. Let $(X, G)$ be a complete $G$-metric space and let $A, B, C, R, S$ and $T$ be six mappings on $X$ into itself satisfying the following conditions

(i) $A(X) \subseteq T(X), B(X) \subseteq S(X), C(X) \subseteq R(X)$.

(ii) $\forall x, y, z \in X$.

$$
\begin{aligned}
G(A x, B y, C z) \leq & \alpha\{G(R x, T y, S z)+G(R x, B y, C z)+G(R x, A x, A x)\} \\
+ & \beta\{\max \{G(T y, B y, C z), G(B y, T y, S z), G(T y, A x, A x), \\
& \quad G(C z, R x, S z)\}\} \\
+ & \gamma\{G(R x, B y, T y)++G(T y, C z, S z)+G(S z, A x, A x)\}
\end{aligned}
$$

where $\alpha, \beta, \gamma>0$ and $6 \alpha+4 \beta+5 \gamma<\frac{1}{2}$

(iii) If one of the following condition is satisfied:

(a) Either $A$ or $R$ is $G$-continuous, the pair $(A, R)$ is weakly commuting, the pair $(B, T)$ and $(C, S)$ are weakly compatible;

(b) Either $B$ or $T$ is $G$-continuous, the pair $(B, T)$ is weakly commuting, the pair $(A, R)$ and $(C, S)$ are weakly compatible; 
(c) Either $C$ or $S$ is $G$-continuous, the pair $(C, S)$ is weakly commuting, the pair $(A, R)$ and $(B, T)$ are weakly compatible;

Then:

(1) One of the pair $(A, R),(B, T)$ or $(C, S)$ has a coincidence point in $X$;

(2) The mappings $A, B, C, S, T$ and $R$ have a unique common fixed point in $X$.

Proof. Suppose the mappings $A, B, C, S, T$ and $R$ satisfy the condition (3.1). Let $x_{0} \in X$ be an arbitrary point. Since $A(X) \subseteq T(X), B(X) \subseteq S(X)$, $C(X) \subseteq R(X)$ then there exist $x_{1}, x_{2}, x_{3} \in X$ such that

$$
A x_{0}=T x_{1}=y_{0}, \quad B x_{1}=S x_{2}=y_{1} \text { and } C x_{2}=R x_{3}=y_{2}
$$

Inductively we can construct a sequence $\left\{y_{n}\right\}$ in $X$ such that $A x_{3 n}=T x_{3 n+1}=y_{3 n}, B x_{3 n+1}=S x_{3 n+2}=y_{3 n+1}$ and $C x_{3 n+2}=R x_{3 n+3}=y_{3 n+2}$ for $n=0,1,2,3 \ldots$

If there exists $n_{0} \in N$ s.t. $y_{n_{0}}=y_{n_{0}+1}$ then the conclusion (1) of Theorem(3.1) holds. In fact if there exists $p \in N$ s.t $y_{3 p+2}=y_{3 p+3}$ then $A u=R u$ where $u=x_{3 p+3}$. Thus the pair $(A, R)$ has a coincidence point $u \in X$. if $y_{3 p}=y_{3 p+1}$ then $B u=T u$ where $u=x_{3 p+1}$. Therefore the pair $(B, T)$ has a coincidence point $u \in X$. if $y_{3 p+1}=y_{3 p+2}$ then $C u=S u$ where $u=x_{3 p+2}$ and so the pair $(C, S)$ has a coincidence point $u \in X$.

On the other hand, if there exists $n_{0} \in N$ s.t. $y_{n_{0}}=y_{n_{0}+1}=y_{n_{0}+2}$ then $y_{n}=y_{n_{0}}$ for any $n \geq n_{0}$. This implies $\left\{y_{n}\right\}$ is a $G$-cauchy sequence. Actually, if there exists $p \in N$ s.t $y_{3 p}=y_{3 p+1}=y_{3 p+2}$ then applying the contractive condition (3.1) with $x=y_{3 p+3}, y=y_{3 p+1}, z=y_{3 p+2}$ we get,

$$
\begin{aligned}
& G\left(y_{3 p+1}, y_{3 p+2}, y_{3 p+3}\right)=G\left(A x_{3 p+3}, B x_{3 p+1}, C x_{3 p+2}\right) \\
& \leq \alpha\left\{G\left(R x_{3 p+3}, T x_{3 p+1}, S x_{3 p+2}\right)+G\left(R x_{3 p+3}, B x_{3 p+1}, C x_{3 p+2}\right)\right. \\
& \left.+G\left(R x_{3 p+3}, A x_{3 p+3}, A x_{3 p+3}\right)\right\}+\beta\left\{\operatorname { m a x } \left\{G\left(T x_{3 p+1}, B x_{3 p+1}, C x_{3 p+2}\right),\right.\right. \\
& G\left(B x_{3 p+1}, T x_{3 p+1}, S x_{3 p+2}\right), G\left(T x_{3 p+1}, A x_{3 p+3}, A x_{3 p+3}\right), \\
& \left.\left.G\left(C x_{3 p+2}, R x_{3 p+3}, S x_{3 p+2}\right)\right\}\right\}+\gamma\left\{G\left(R x_{3 p+3}, B x_{3 p+1}, T x_{3 p+1}\right)+\right. \\
& \left.G\left(T x_{3 p+1}, C x_{3 p+2}, S x_{3 p+2}\right)+G\left(S x_{3 p+2}, A x_{3 p+3}, A x_{3 p+3}\right)\right\} \\
& \leq \alpha\left\{G\left(y_{3 p+2}, y_{3 p}, y_{3 p+1}\right)+G\left(y_{3 p+2}, y_{3 p+1}, y_{3 p+2}\right)+G\left(y_{3 p+2}, y_{3 p+3}, y_{3 p+3}\right)\right\}+ \\
& \beta\left\{\operatorname { m a x } \left\{G\left(y_{3 p}, y_{3 p+1}, y_{3 p+2}\right), G\left(y_{3 p+1}, y_{3 p}, y_{3 p+1}\right), G\left(y_{3 p}, y_{3 p+3}, y_{3 p+3}\right)\right.\right. \\
& \left.\left.G\left(y_{3 p+2}, y_{3 p+2}, y_{3 p+1}\right)\right\}\right\}+\gamma\left\{G\left(y_{3 p+2}, y_{3 p+1}, y_{3 p}\right)+G\left(y_{3 p}, y_{3 p+2}, y_{3 p+1}\right)+\right. \\
& \left.G\left(y_{3 p+1}, y_{3 p+3}, y_{3 p+3}\right)\right\}
\end{aligned}
$$




$$
\begin{aligned}
& \leq \alpha\left\{0+0+G\left(y_{3 p+2}, y_{3 p+3}, y_{3 p+3}\right)\right\}+\beta\left\{\max \left\{0,0, G\left(y_{3 p}, y_{3 p+3}, y_{3 p+3}\right), 0\right\}\right\} \\
& +\gamma\left\{0,0, G\left(y_{3 p+1}, y_{3 p+3}, y_{3 p+3}\right)\right\}
\end{aligned}
$$

This implies that

$$
G\left(y_{3 p+1}, y_{3 p+2}, y_{3 p+3}\right) \leq(\alpha+\beta+\gamma) G\left(y_{3 p+2}, y_{3 p+3}, y_{3 p+3}\right)
$$

If $y_{3 p+3} \neq y_{3 p+1}$ then from $G(3), G(4)$ and Proposition(2.4)(iii), we get

$$
\begin{aligned}
0<G\left(y_{3 p+1}, y_{3 p+2}, y_{3 p+3}\right) & \leq 2(\alpha+\beta+\gamma) G\left(y_{3 p+2}, y_{3 p+2}, y_{3 p+3}\right) \\
& \leq 2(\alpha+\beta+\gamma) G\left(y_{3 p+1}, y_{3 p+2}, y_{3 p+3}\right)
\end{aligned}
$$

This implies that $\alpha+\beta+\gamma \geq \frac{1}{2}$ which is contradiction as $6 \alpha+4 \beta+5 \gamma<\frac{1}{2}$.

So we find $y_{n}=y_{3 p}$ for any $n \geq 3 p$.

This implies that $\left\{y_{n}\right\}$ is a $G$-cauchy sequence.

Similarily we can show that $\left\{y_{n}\right\}$ is a $G$-cauchy sequence for $y_{3 p+1}=y_{3 p+2}=$ $y_{3 p+3}$ or $y_{3 p+2}=y_{3 p+3}=y_{3 p+4}$ for some $p \in N$.

For the rest of the paper assume that $y_{n} \neq y_{m}$ for any $n \neq m$.

Put $x=y_{3 n}, y=y_{3 n+1}, z=y_{3 n+2}$ in inquality(3.1) and using the condition $G(3), G(4), G(5)$ and Proposition(2.4)(iv)we get

$$
\begin{aligned}
& G\left(y_{3 n}, y_{3 n+1}, y_{3 n+2}\right)=G\left(A x_{3 n}, B x_{3 n+1}, C x_{3 n+2}\right) \\
& \leq \alpha\left\{G\left(R x_{3 n}, T x_{3 n+1}, S x_{3 n+2}\right)+G\left(R x_{3 n}, B x_{3 n+1}, C x_{3 n+2}\right)\right. \\
& \left.+G\left(R x_{3 n}, A x_{3 n}, A x_{3 n}\right)\right\}+\beta\left\{\operatorname { m a x } \left\{G\left(T x_{3 n+1}, B x_{3 n+1}, C x_{3 n+2}\right)\right.\right. \\
& G\left(B x_{3 n+1}, T x_{3 n+1}, S x_{3 n+2}\right), G\left(T x_{3 n+1}, A x_{3 n}, A x_{3 n}\right) \\
& \left.\left.G\left(C x_{3 n+2}, R x_{3 n}, S x_{3 n+2}\right)\right\}\right\}+\gamma\left\{G\left(R x_{3 n}, B x_{3 n+1}, T x_{3 n+1}\right)+\right. \\
& \left.G\left(T x_{3 n+1}, C x_{3 n+2}, S x_{3 n+2}\right)+G\left(S x_{3 n+2}, A x_{3 n}, A x_{3 n}\right)\right\} \\
& \leq \alpha\left\{G\left(y_{3 n-1}, y_{3 n}, y_{3 n+1}\right)+G\left(y_{3 n-1}, y_{3 n+1}, y_{3 n+2}\right)+G\left(y_{3 n-1}, y_{3 n}, y_{3 n}\right)\right\}+ \\
& \beta\left\{m a x \left\{G\left(y_{3 n}, y_{3 n+1}, y_{3 n+2}\right), G\left(y_{3 n+1}, y_{3 n}, y_{3 n+1}\right), G\left(y_{3 n}, y_{3 n}, y_{3 n}\right)\right.\right. \\
& \left.\left.G\left(y_{3 n+2}, y_{3 n-1}, y_{3 n+1}\right)\right\}\right\}+\gamma\left\{G\left(y_{3 n-1}, y_{3 n+1}, y_{3 n}\right)+G\left(y_{3 n}, y_{3 n+2}, y_{3 n+1}\right)+\right. \\
& \left.G\left(y_{3 n+1}, y_{3 n}, y_{3 n}\right)\right\} \\
& \leq \alpha\left\{G\left(y_{3 n-1}, y_{3 n}, y_{3 n+1}\right)+G\left(y_{3 n-1}, y_{3 n}, y_{3 n+1}\right)+2 G\left(y_{3 n}, y_{3 n+1}, y_{3 n+2}\right)+\right. \\
& G\left(y_{3 n-1}, y_{3 n}, y_{3 n+1}\right\}+\beta\left\{\operatorname { m a x } \left\{G\left(y_{3 n}, y_{3 n+1}, y_{3 n+2}\right), G\left(y_{3 n-1}, y_{3 n}, y_{3 n+1}\right),\right.\right. \\
& \left.\left.0, G\left(y_{3 n-1}, y_{3 n}, y_{3 n+1}\right)+2 G\left(y_{3 n}, y_{3 n+1}, y_{3 n+2}\right)\right\}\right\}+\gamma\left\{G\left(y_{3 n-1}, y_{3 n}, y_{3 n+1}\right)+\right. \\
& \left.G\left(y_{3 n}, y_{3 n+1}, y_{3 n+2}\right)+G\left(y_{3 n-1}, y_{3 n}, y_{3 n+1}\right)\right\} \\
& \leq \alpha\left\{3 G\left(y_{3 n-1}, y_{3 n}, y_{3 n+1}\right)+2 G\left(y_{3 n}, y_{3 n+1}, y_{3 n+2}\right)\right\}+ \\
& \beta\left\{G\left(y_{3 n-1}, y_{3 n}, y_{3 n+1}\right)+2 G\left(y_{3 n}, y_{3 n+1}, y_{3 n+2}\right)\right\}+ \\
& \gamma\left\{2 G\left(y_{3 n-1}, y_{3 n}, y_{3 n+1}\right)+G\left(y_{3 n}, y_{3 n+1}, y_{3 n+2}\right)\right\}
\end{aligned}
$$


Now we show that $G\left(y_{3 n}, y_{3 n+1}, y_{3 n+2}\right) \leq G\left(y_{3 n-1}, y_{3 n}, y_{3 n+1}\right)$ for every $n \in N$. If $G\left(y_{3 n}, y_{3 n+1}, y_{3 n+2}\right)>G\left(y_{3 n-1}, y_{3 n}, y_{3 n+1}\right)$ for some $n \in N$ then by above we have

$$
\begin{aligned}
G\left(y_{3 n}, y_{3 n+1}, y_{3 n+2}\right) & <5 \alpha\left\{G\left(y_{3 n}, y_{3 n+1}, y_{3 n+2}\right)\right\}+3 \beta\left\{G\left(y_{3 n}, y_{3 n+1}, y_{3 n+2}\right)\right\}+ \\
& 3 \gamma\left\{G\left(y_{3 n}, y_{3 n+1}, y_{3 n+2}\right)\right\} \\
& <(5 \alpha+3 \beta+3 \gamma)\left\{G\left(y_{3 n}, y_{3 n+1}, y_{3 n+2}\right)\right\}
\end{aligned}
$$

This implies that $5 \alpha+3 \beta+3 \gamma>1>\frac{1}{2}$ which is contradiction as $6 \alpha+4 \beta+5 \gamma<\frac{1}{2}$ Hence we get $G\left(y_{3 n}, y_{3 n+1}, y_{3 n+2}\right) \leq G\left(y_{3 n-1}, y_{3 n}, y_{3 n+1}\right)$ for every $n \in N$.

This implies that

$$
\begin{aligned}
& G\left(y_{3 n}, y_{3 n+1}, y_{3 n+2}\right) \leq 5 \alpha\left\{G\left(y_{3 n-1}, y_{3 n}, y_{3 n+1}\right)\right\}+3 \beta\left\{G\left(y_{3 n-1}, y_{3 n}, y_{3 n+1}\right)\right\}+ \\
& \gamma\left\{G\left(y_{3 n-1}, y_{3 n}, y_{3 n+1}\right)\right\} \\
& \leq(5 \alpha+3 \beta+3 \gamma)\left\{G\left(y_{3 n-1}, y_{3 n}, y_{3 n+1}\right)\right\} \\
&<(6 \alpha+4 \beta+5 \gamma)\left\{G\left(y_{3 n-1}, y_{3 n}, y_{3 n+1}\right)\right\} \\
& \Rightarrow G\left(y_{3 n}, y_{3 n+1}, y_{3 n+2}\right) \leq q .\left\{G\left(y_{3 n-1}, y_{3 n}, y_{3 n+1}\right)\right\}
\end{aligned}
$$

where $q=6 \alpha+4 \beta+5 \gamma$

Similarily we can show that

$$
G\left(y_{3 n+1}, y_{3 n+2}, y_{3 n+3}\right)<q .\left\{G\left(y_{3 n}, y_{3 n+1}, y_{3 n+2}\right)\right\}
$$

Thus it follows from inequality (3.2) and (3.3) that for all $n \in N$

$$
\begin{aligned}
G\left(y_{n}, y_{n+1}, y_{n+2}\right)<q\left\{G\left(y_{n-1}, y_{n}, y_{n+1}\right)\right\}<q^{2} \cdot\left\{G\left(y_{n-2}, y_{n-1}, y_{n}\right)\right\} \\
<\ldots<q^{n}\left\{G\left(y_{0}, y_{1}, y_{2}\right)\right\}
\end{aligned}
$$

Therefore for all $n, m \in N, n<m$, by $G(3)$ and $G(5)$ we have

$$
\begin{aligned}
G\left(y_{n}, y_{m}, y_{m}\right) & \leq G\left(y_{n}, y_{n+1}, y_{n+1}\right)+G\left(y_{n+1}, y_{n+2}, y_{n+2}\right)+G\left(y_{n+1}, y_{n+2}, y_{n+2}\right)+ \\
& \ldots+G\left(y_{m-1}, y_{m}, y_{m}\right) \\
& <q^{n} G\left(y_{0}, y_{1}, y_{2}\right)+q^{n+1} G\left(y_{0}, y_{1}, y_{2}\right)+\ldots+q^{m-1} G\left(y_{0}, y_{1}, y_{2}\right) \\
& <\left(q^{n}+q^{n+1}+q^{n+2}+\ldots+q^{m-1}\right) G\left(y_{0}, y_{1}, y_{2}\right) \\
& <\frac{q^{n}}{1-q} G\left(y_{0}, y_{1}, y_{2}\right) \rightarrow 0
\end{aligned}
$$

as $n \rightarrow \infty$. Hence $\left\{y_{n}\right\}$ is a $G$-cauchy sequence in $X$.

Since $X$ is complete $G$-metric space, then there exists a point $u \in X$ s.t. $y_{n} \rightarrow u$ as $n \rightarrow \infty$. 
Since the sequences $\left\{A x_{3 n}\right\}=\left\{T x_{3 n+1}\right\},\left\{B x_{3 n+1}\right\}=\left\{S x_{3 n+2}\right\}$ and $\left\{C x_{3 n-1}\right\}=\left\{R x_{3 n}\right\}$ are also the subsequences of $\left\{y_{n}\right\}$, then they all converges to $u$.

Hence as $n \rightarrow \infty$

$$
\begin{aligned}
& y_{3 n}=A x_{3 n}=T x_{3 n+1} \rightarrow u \\
& y_{3 n+1}=B x_{3 n+1}=S x_{3 n+2} \rightarrow u \\
& y_{3 n-1}=C x_{3 n-1}=R x_{3 n} \rightarrow u
\end{aligned}
$$

Now we prove that $u$ is the common fixed point of $A, B, C, R, S$ and $T$ under condition $(a)$.

Case I. First suppose that $R$ is continuous, the pair $(A, R)$ is weakly commuting, the pairs $(B, T)$ and $(C, S)$ is weakly compatible. First we will prove that $u=A u=R u$.

By equation(3.4) and weakly commuting of the pair $(A, R)$, we have as $n \rightarrow \infty$

$$
G\left(A R x_{3 n}, R A x_{3 n}, R A x_{3 n}\right) \leq G\left(A x_{3 n}, R x_{3 n}, R x_{3 n}\right) \rightarrow 0
$$

Since $R$ is continuous then by inequality(3.5) $R^{2} x_{3 n} \rightarrow R u, R A x_{3 n} \rightarrow R u$ as $n \rightarrow \infty$.

We know that $A R x_{3 n} \rightarrow R u$ then from inquality (3.1) we have

$$
\begin{aligned}
& G\left(A R x_{3 n}, B x_{3 n+1}, C x_{3 n+2}\right) \leq \alpha\left\{G\left(R^{2} x_{3 n}, T x_{3 n+1}, S x_{3 n+2}\right)\right. \\
& +G\left(R^{2} x_{3 n}, B x_{3 n+1}, C x_{3 n+2}\right)+ \\
& \left.G\left(R^{2} x_{3 n}, A R x_{3 n}, A R x_{3 n}\right)\right\}+\beta\left\{\operatorname { m a x } \left\{G\left(T x_{3 n+1}, B x_{3 n+1}, C x_{3 n+2}\right),\right.\right. \\
& G\left(B x_{3 n+1}, T x_{3 n+1}, S x_{3 n+2}\right), G\left(T x_{3 n+1}, A R x_{3 n}, A R x_{3 n}\right), \\
& \left.\left.G\left(C x_{3 n+2}, R^{2} x_{3 n}, S x_{3 n+2}\right)\right\}\right\}+\gamma\left\{G\left(R^{2} x_{3 n}, B x_{3 n+1}, T x_{3 n+1}\right)+\right. \\
& \left.G\left(T x_{3 n+1}, C x_{3 n+2}, S x_{3 n+2}\right)+G\left(S x_{3 n+2}, A R x_{3 n}, A R x_{3 n}\right)\right\}
\end{aligned}
$$

Taking $n \rightarrow \infty$

$$
\begin{aligned}
& G(R u, u, u) \leq \alpha\{G(R u, u, u)+G(R u, u, u)+G(R u, R u, R u)\} \\
& +\beta\{\max \{G(u, u, u), G(u, u, u), \\
& G(u, R u, R u), G(u, R u, u)\}\}+\gamma\{G(R u, u, u)+G(u, u, u)+G(u, R u, R u)\} \\
& \leq \alpha\{2 G(R u, u, u)+0\}+\beta\{\max \{0,0, G(u, R u, R u), G(u, R u, u)\}\}+ \\
& \gamma\{G(R u, u, u)+0+G(u, R u, R u)\} \\
& \leq 2 \alpha G(R u, u, u)+2 \beta G(R u, u, u)+3 \gamma G(R u, u, u)
\end{aligned}
$$




$$
\Rightarrow G(R u, u, u) \leq(2 \alpha+2 \beta+3 \gamma) G(R u, u, u)
$$

which implies that $G(R u, u, u)=0$, so that $R u=u$ as $6 \alpha+4 \beta+5 \gamma<\frac{1}{2}$.

Again using inequality(3.1) we have

$G\left(A u, B x_{3 n+1}, C x_{3 n+2}\right) \leq \alpha\left\{G\left(R u, T x_{3 n+1}, S x_{3 n+2}\right)+G\left(R u, B x_{3 n+1}, C x_{3 n+2}\right)+\right.$ $G(R u, A u, A u)\}+\beta\left\{\max \left\{G\left(T x_{3 n+1}, B x_{3 n+1}, C x_{3 n+2}\right)\right.\right.$,

$G\left(B x_{3 n+1}, T x_{3 n+1}, S x_{3 n+2}\right), G\left(T x_{3 n+1}, A u, A u\right)$,

$\left.\left.G\left(C x_{3 n+2}, R u, S x_{3 n+2}\right)\right\}\right\}+\gamma\left\{G\left(R u, B x_{3 n+1}, T x_{3 n+1}\right)+\right.$

$\left.G\left(T x_{3 n+1}, C x_{3 n+2}, S x_{3 n+2}\right)+G\left(S x_{3 n+2}, A u, A u\right)\right\}$

Taking $n \rightarrow \infty$ and $u=R u$, we have

$$
\begin{gathered}
G(A u, u, u) \leq \alpha\{G(u, u, u)+G(u, u, u)+G(u, A u, A u)\} \\
+\beta\{\max \{G(u, u, u), G(u, u, u) \\
G(u, A u, A u), G(u, u, u)\}\}+\gamma\{G(u, u, u)+G(u, u, u)+G(u, A u, A u)\} \\
\leq \alpha\{2 G(A u, u, u)\}+\beta\{2 G(A u, u, u)\}+\gamma\{2 G(A u, u, u)\} \\
\Rightarrow G(A u, u, u) \leq(2 \alpha+2 \beta+2 \gamma) G(A u, u, u)
\end{gathered}
$$

which implies that $G(A u, u, u)=0$, so that $A u=u$.

Thus we have $u=A u=R u$. Hence $u$ is the common fixed point of $A$ and $R$.

Again we show that $u$ is the common fixed point of $B$ and $T$.

Since $A(X) \subseteq T(X)$ and $u=A u \in A(X)$, there exists a point $v \in X$ s.t. $u=A u=T v$.

Now by inequality(3.1) we have

$$
\begin{aligned}
& G\left(A u, B v, C x_{3 n+2}\right) \leq \alpha\left\{G\left(R u, T v, S x_{3 n+2}\right)+G\left(R u, B v, C x_{3 n+2}\right)\right. \\
& +G(R u, A u, A u)\}+ \\
& \beta\left\{\operatorname { m a x } \left\{G\left(T v, B v, C x_{3 n+2}\right), G\left(B v, T v, S x_{3 n+2}\right), G(T v, A u, A u),\right.\right. \\
& \left.\left.G\left(C x_{3 n+2}, R u, S x_{3 n+2}\right)\right\}\right\}+\gamma\{G(R u, B v, T v)+ \\
& \left.G\left(T v, C x_{3 n+2}, S x_{3 n+2}\right)+G\left(S x_{3 n+2}, A u, A u\right)\right\}
\end{aligned}
$$

Taking $n \rightarrow \infty$ and using $u=A u=R u=T v$, we get

$$
\begin{aligned}
& G(u, B v, u) \leq \alpha\{G(u, u, u)+G(u, B v, u)+G(u, u, u)\} \\
& +\beta\{\max \{G(u, B v, u), G(B v, u, u) \\
& G(u, u, u), G(u, u, u)\}\}+\gamma\{G(u, B v, u)+G(u, u, u)+G(u, u, u)\} \\
& \leq(\alpha+\beta+\gamma) G(u, B v, u)
\end{aligned}
$$


This implies that $G(u, B v, u)=0 \Rightarrow u=B v$.

Also $T v=u \Rightarrow T v=u=B v$. $T u$.

Since the pair $(B, T)$ is weakly compatible, we have $B u=B T v=T B v=$

Again using inequality(3.1) we have

$$
\begin{aligned}
& G\left(A u, B u, C x_{3 n+2}\right) \leq \alpha\left\{G\left(R u, T u, S x_{3 n+2}\right)+G\left(R u, B u, C x_{3 n+2}\right)\right. \\
& +G(R u, A u, A u)\}+ \\
& \beta\left\{\operatorname { m a x } \left\{G\left(T u, B u, C x_{3 n+2}\right), G\left(B u, T u, S x_{3 n+2}\right), G(T u, A u, A u),\right.\right. \\
& \left.\left.G\left(C x_{3 n+2}, R u, S x_{3 n+2}\right)\right\}\right\}+\gamma\{G(R u, B u, T u)+ \\
& \left.G\left(T u, C x_{3 n+2}, S x_{3 n+2}\right)+G\left(S x_{3 n+2}, A u, A u\right)\right\}
\end{aligned}
$$

Taking $n \rightarrow \infty$, using $u=A u=R u$ and $B u=T u$, we get

$$
\begin{aligned}
& G(u, B u, u) \leq \alpha\{G(u, B u, u)+G(u, B u, u)+G(u, u, u)\} \\
& +\beta\{\max \{G(B u, B u, u), \\
& G(B u, B u, u), G(B u, u, u), G(u, u, u)\}\}+\gamma\{G(u, B u, B u)+ \\
& G(B u, u, u)+G(u, u, u)\} \\
& \leq(2 \alpha+2 \beta+2 \gamma) G(u, B u, u)
\end{aligned}
$$

This implies that $G(u, B u, u)=0 \Rightarrow u=B u$.

Also $T u=B u \Rightarrow T u=u=B u$.

This shows that $u$ is a common fixed point of $B$ and $T$.

Now we show that $u$ is a common fixed point of $C$ and $S$.

Since $B(X) \subseteq S(X)$ and $u=B u \in B(X)$, therefore there exists a point $w \in X$ s.t. $u=B u=S w$.

Using the inequality(3.1) we have

$$
\begin{aligned}
& G(A u, B u, C w) \leq \alpha\{G(R u, T u, S w)+G(R u, B u, C w)+G(R u, A u, A u)\}+ \\
& \beta\{\max \{G(T u, B u, C w), G(B u, T u, S w), G(T u, A u, A u), G(C w, R u, S w)\}\}+ \\
& \gamma\{G(R u, B u, T u)+G(T u, C w, S w)+G(S w, A u, A u)\}
\end{aligned}
$$

using $u=A u=R u=B u=T u=S w$, we get

$$
\begin{aligned}
G(u, u, C w) & \leq \alpha\{G(u, u, u)+G(u, u, C w)+G(u, u, u)\}+ \\
& \beta\{\max \{G(u, u, C w), G(u, u, u), G(u, u, u), G(C w, u, u)\}\}+ \\
& \gamma\{G(u, u, u)+G(u, C w, u)+G(u, u, u)\} \\
& \leq(\alpha+\beta+\gamma) G(u, u, C w)
\end{aligned}
$$


This implies that $G(u, u, C w)=0 \Rightarrow u=C w$.

Also $u=S w \Rightarrow S w=u=C w$. Su.

Since the pair $(C, S)$ is weakly compatible, we have $C u=C S w=S C w=$

Again using inequality(3.1) we have

$$
\begin{aligned}
& G(A u, B u, C u) \leq \alpha\{G(R u, T u, S u)+G(R u, B u, C u)+G(R u, A u, A u)\}+ \\
& \beta\{\max \{G(T u, B u, C u), G(B u, T u, S u), G(T u, A u, A u), G(C u, R u, S u)\}\}+ \\
& \gamma\{G(R u, B u, T u)+G(T u, C u, S u)+G(S u, A u, A u)\}
\end{aligned}
$$

using $u=A u=R u=B u=T u$ and $C u=S u$, we get

$$
\begin{aligned}
G(u, u, C u) & \leq \alpha\{G(u, u, C u)+G(u, u, C u)+G(u, u, u)\}+ \\
& \beta\{\max \{G(u, u, C u), G(u, u, C u), G(u, u, u), G(C u, u, C u)\}\}+ \\
& \gamma\{G(u, u, u)+G(u, C u, C u)+G(C u, u, u)\} \\
& \leq(2 \alpha+2 \beta+3 \gamma) G(u, u, C u)
\end{aligned}
$$

This implies that $G(u, u, C u)=0 \Rightarrow u=C u$.

Also $S u=C u$, therefore we get $C u=u=S u$.

This shows that $u$ is a common fixed point of $C$ and $S$.

Hence $u$ is a common fixed point of $A, B, C, R, S$ and $T$.

Case II. Suppose that $A$ is continuous, the pair $(A, R)$ is weakly commuting, the pairs $(B, T)$ and $(C, S)$ are weakly compatible.

First we prove that $u=A u$.

Since the pair $(A, R)$ is weakly commuting, then by inequality $(3.4)$ we have as $n \rightarrow \infty$

$$
G\left(A R x_{3 n}\right), R A x_{3 n}, R A x_{3 n} \leq G\left(A x_{3 n}\right), R x_{3 n}, R x_{3 n} \rightarrow 0 .
$$

Since $A$ is continuous then $A^{2} x_{3 n} \rightarrow A u$, as $n \rightarrow \infty$.

By inequality(3.5)we have $R A x_{3 n} \rightarrow A u$, as $n \rightarrow \infty$.

From inequality (3.1) we have

$$
\begin{aligned}
& G\left(A^{2} x_{3 n}, B x_{3 n+1}, C x_{3 n+2}\right) \leq \alpha\left\{G\left(R A x_{3 n}, T x_{3 n+1}, S x_{3 n+2}\right)\right. \\
& +G\left(R A x_{3 n}, B x_{3 n+1}, C x_{3 n+2}\right)+ \\
& \left.G\left(R A x_{3 n}, A^{2} x_{3 n}, A^{2} x_{3 n}\right)\right\}+\beta\left\{\operatorname { m a x } \left\{G\left(T x_{3 n+1}, B x_{3 n+1}, C x_{3 n+2}\right),\right.\right. \\
& G\left(B x_{3 n+1}, T x_{3 n+1}, S x_{3 n+2}\right), G\left(T x_{3 n+1}, A^{2} x_{3 n}, A^{2} x_{3 n}\right), \\
& \left.\left.G\left(C x_{3 n+2}, R A x_{3 n}, S x_{3 n+2}\right)\right\}\right\}+\gamma\left\{G\left(R A x_{3 n}, B x_{3 n+1}, T x_{3 n+1}\right)+\right. \\
& \left.G\left(T x_{3 n+1}, C x_{3 n+2}, S x_{3 n+2}\right)+G\left(S x_{3 n+2}, A^{2} x_{3 n}, A^{2} x_{3 n}\right)\right\}
\end{aligned}
$$


Taking $n \rightarrow \infty$

$$
\begin{aligned}
G(A u, u, u) & \leq \alpha\{G(A u, u, u)+G(A u, u, u)+G(A u, A u, A u)\} \\
& +\beta\{\max \{G(u, u, u), G(u, u, u) \\
& , G(u, A u, A u), G(u, A u, u)\}\}+\gamma\{G(A u, u, u) \\
& +G(u, u, u)+G(u, A u, A u)\} \\
& \leq 2 \alpha G(A u, u, u)+2 \beta G(A u, u, u)+3 \gamma G(A u, u, u) \\
& \leq(2 \alpha+2 \beta+3 \gamma) G(A u, u, u)
\end{aligned}
$$

which implies that $G(A u, u, u)=0$, so that $A u=u$.

Now we prove that $u$ is the common fixed point of $B$ and $T$.

Since $A(X) \subseteq T(X)$ and $u=A u \in A(X)$, there exists a point $v \in X$ s.t. $u=A u=T v$.

Now by inequality(3.1) we have

$$
\begin{aligned}
& G\left(A^{2} x_{3 n}, B v, C x_{3 n+2}\right) \leq \alpha\left\{G\left(R A x_{3 n}, T v, S x_{3 n+2}\right)+G\left(R A x_{3 n}, B v, C x_{3 n+2}\right)+\right. \\
& \left.G\left(R A x_{3 n}, A^{2} x_{3 n}, A^{2} x_{3 n}\right)\right\}+\beta\left\{\operatorname { m a x } \left\{G\left(T v, B v, C x_{3 n+2}\right),\right.\right. \\
& G\left(B v, T v, S x_{3 n+2}\right), G\left(T v, A^{2} x_{3 n}, A^{2} x_{3 n}\right), \\
& \left.\left.G\left(C x_{3 n+2}, R A x_{3 n}, S x_{3 n+2}\right)\right\}\right\}+\gamma\left\{G\left(R A x_{3 n}, B v, T v\right)+\right. \\
& \left.G\left(T v, C x_{3 n+2}, S x_{3 n+2}\right)+G\left(S x_{3 n+2}, A^{2} x_{3 n}, A^{2} x_{3 n}\right)\right\}
\end{aligned}
$$

Taking $n \rightarrow \infty$ and using $u=A u=T v$, we get

$$
\begin{aligned}
G(u, B v, u) & \leq \alpha\{G(u, u, u)+G(u, B v, u)+G(u, u, u)\} \\
& +\beta\{\max \{G(u, B v, u), G(B v, u, u), \\
& G(u, u, u), G(u, u, u)\}\}+\gamma\{G(u, B v, u)+G(u, u, u)+G(u, u, u)\} \\
& \leq(\alpha+\beta+\gamma) G(u, B v, u)
\end{aligned}
$$

This implies that $G(u, B v, u)=0 \Rightarrow u=B v$.

Also $T v=u \Rightarrow T v=u=B v$. $T u$.

Since the pair $(B, T)$ is weakly compatible, we have $B u=B T v=T B v=$

Again using inequality(3.1) we have

$$
\begin{aligned}
G\left(A x_{3 n}, B u, C x_{3 n+2}\right) & \leq \alpha\left\{G\left(R x_{3 n}, T u, S x_{3 n+2}\right)+G\left(R x_{3 n}, B u, C x_{3 n+2}\right)+\right. \\
& \left.G\left(R x_{3 n}, A x_{3 n}, A x_{3 n}\right)\right\}+\beta\left\{\operatorname { m a x } \left\{G\left(T u, B u, C x_{3 n+2}\right),\right.\right. \\
& G\left(B u, T u, S x_{3 n+2}\right), G\left(T u, A x_{3 n}, A x_{3 n}\right), \\
& \left.\left.G\left(C x_{3 n+2}, R x_{3 n}, S x_{3 n+2}\right)\right\}\right\}+\gamma\left\{G\left(R x_{3 n}, B u, T u\right)+\right. \\
& \left.G\left(T u, C x_{3 n+2}, S x_{3 n+2}\right)+G\left(S x_{3 n+2}, A x_{3 n}, A x_{3 n}\right)\right\}
\end{aligned}
$$


Taking $n \rightarrow \infty$, using $u=A u=R u$ and $B u=T u$, we get

$$
\begin{aligned}
G(u, B u, u) & \leq \alpha\{G(u, B u, u)+G(u, B u, u)+G(u, u, u)\} \\
& +\beta\{\max \{G(B u, B u, u), G(B u, B u, u) \\
& G(B u, u, u), G(u, u, u)\}\}+\gamma\{G(u, B u, B u) \\
& +G(B u, u, u)+G(u, u, u)\} \\
& \leq(2 \alpha+2 \beta+3 \gamma) G(u, B u, u)
\end{aligned}
$$

This implies that $G(u, B u, u)=0 \Rightarrow u=B u$.

Also $T u=B u \Rightarrow T u=u=B u$.

Thus $u$ is a common fixed point of $B$ and $T$.

Now we show that $u$ is a common fixed point of $C$ and $S$.

Since $B(X) \subseteq S(X)$ and $u=B u \in B(X)$, therefore there exists a point $w \in X$ s.t. $u=B u=S w$.

Using the inequality(3.1) we have

$$
\begin{aligned}
G\left(A x_{3 n}, B u, C w\right) & \leq \alpha\left\{G\left(R x_{3 n}, T u, S w\right)+G\left(R x_{3 n}, B u, C w\right)\right. \\
& \left.+G\left(R x_{3 n}+A x_{3 n}, A x_{3 n}\right)\right\}+ \\
& \beta\left\{\operatorname { m a x } \left\{G(T u, B u, C w), G(B u, T u, S w), G\left(T u, A x_{3 n}, A x_{3 n}\right),\right.\right. \\
& \left.\left.G\left(C w, R x_{3 n}, S w\right)\right\}\right\}+\gamma\left\{G\left(R x_{3 n}, B u, T u\right)\right. \\
& +G(T u, C w, S w)+ \\
& \left.G\left(S w, A x_{3 n}, A x_{3 n}\right)\right\}
\end{aligned}
$$

Taking $n \rightarrow \infty$ and using $u=B u=T u=S w$, we get

$$
\begin{aligned}
G(u, u, C w) & \leq \alpha\{G(u, u, u)+G(u, u, C w)+G(u, u, u)\}+ \\
& \beta\{\max \{G(u, u, C w), G(u, u, u), G(u, u, u), G(C w, u, u)\}\}+ \\
& \gamma\{G(u, u, u)+G(u, C w, u)+G(u, u, u)\} \\
& \leq(\alpha+\beta+\gamma) G(u, u, C w)
\end{aligned}
$$

This implies that $G(u, u, C w)=0 \Rightarrow u=C w$.

Also $u=S w \Rightarrow S w=u=C w$.

Since the pair $(C, S)$ is weakly compatible, we have $C u=C S w=S C w=$ $S u$. 
Again using inequality(3.1) we have

$$
\begin{aligned}
G\left(A x_{3 n}, B u, C u\right) & \leq \alpha\left\{G\left(R x_{3 n}, T u, S u\right)+G\left(R x_{3 n}, B u, C u\right)\right. \\
& \left.+G\left(R x_{3 n}, A x_{3 n}, A x_{3 n}\right)\right\}+ \\
& \beta\left\{\operatorname { m a x } \left\{G(T u, B u, C u), G(B u, T u, S u), G\left(T u, A x_{3 n}, A x_{3 n}\right),\right.\right. \\
& \left.\left.G\left(C u, R x_{3 n}, S u\right)\right\}\right\}+\gamma\left\{G\left(R x_{3 n}, B u, T u\right)+G(T u, C u, S u)+\right. \\
& \left.G\left(S u, A x_{3 n}, A x_{3 n}\right)\right\}
\end{aligned}
$$

Taking $n \rightarrow \infty$, using $u=B u=T u$ and $C u=S u$, we get

$$
\begin{aligned}
G(u, u, C u) & \leq \alpha\{G(u, u, C u)+G(u, u, C u)+G(u, u, u)\}+ \\
& \beta\{\max \{G(u, u, C u), G(u, u, C u), G(u, u, u), G(C u, u, C u)\}\}+ \\
& \gamma\{G(u, u, u)+G(u, C u, C u)+G(C u, u, u)\} \\
& \leq(2 \alpha+2 \beta+3 \gamma) G(u, u, C u)
\end{aligned}
$$

This implies that $G(u, u, C u)=0 \Rightarrow u=C u$.

Also $S u=C u$, therefore we get $C u=u=S u$.

This shows that $u$ is a common fixed point of $C$ and $S$.

Finally we will show that $u=R u$.

Since $C(X) \subseteq R(X)$ and $u=C u \in C(X)$, therefore there exists a point $t \in X$ s.t. $u=u=R t$.

Using the inequality(3.1) we have

$$
\begin{aligned}
G(A t, B u, C u) & \leq \alpha\{G(R t, T u, S u)+G(R t, B u, C u)+G(R t, A t, A t)\}+ \\
& \beta\{\max \{G(T u, B u, C u), G(B u, T u, S u), \\
& G(T u, A t, A t), G(C u, R t, S u)\}\}+ \\
& \gamma\{G(R t, B u, T u)+G(T u, C u, S u)+G(S u, A t, A t)\} \\
& \leq \alpha\{G(u, u, u)+G(u, u, u)+G(u, A t, A t)\}+\beta\{\max \{G(u, u, u), \\
& G(u, u, u), G(u, A t, A t), G(u, u, u)\}\}+\gamma\{G(u, u, u) \\
& +G(u, u, u)+G(u, A t, A t)\} \\
& \leq(2 \alpha+2 \beta+2 \gamma) G(A t, u, u)
\end{aligned}
$$

This implies that $G(A t, u, u)=0 \Rightarrow u=A t$.

Since the pair $(A, R)$ is weakly commuting, we have $A u=A R t=R A t=$ $R u=u$.

This shows that $u$ is a common fixed point of $A$ and $R$.

Hence $u$ is a common fixed point of $A, B, C, R, S$ and $T$ when $A$ is continuous and the pair $(A, R)$ is weakly commuting, the pairs $(B, T)$ and $(C, S)$ is weakly compatible. 
Similarily we can prove that the $u$ is a common fixed point of $A, B, C, R, S$ and $T$ when the condition $(b)$ and $(c)$ are hold.

Uniqueness. Let $u$ and $z$ be two common fixed point of $A, B, C, R, S$ and $T$. Using inequality(3.1) we have

$$
\begin{aligned}
G(z, u, u) & \leq \alpha\{G(R z, T u, S u)+G(R z, B u, C u)+G(R z, A z, A z)\}+ \\
& \beta\{\max \{G(T u, B u, C u), G(B u, T u, S u), \\
& G(T u, A z, A z), G(C u, R z, S u)\}\}+ \\
& \gamma\{G(R z, B u, T u)+G(T u, C u, S u)+G(S u, A z, A z)\} \\
& \leq \alpha\{G(z, u, u)+G(z, u, u)+G(z, z, z)\}+\beta\{\max \{G(u, u, u), G(u, u, u), \\
& G(u, z, z), G(u, z, u)\}\}+\gamma\{G(z, u, u)+G(u, u, u)+G(u, z, z)\} \\
& \leq(2 \alpha+2 \beta+3 \gamma) G(z, u, u)
\end{aligned}
$$

This implies that $G(z, u, u)=0 \Rightarrow z=u$.

Thus the common fixed point is unique.

This completes the proof.

In Theorem(3.1), if we take $R=S=T=I$ then following Corollary is obtained.

Corollary 3.1. Let $(X, G)$ be a complete $G$-metric space and let $A, B$ and $C$ be three mappings of $X$ into itself satisfying the following conditions,

$$
\begin{aligned}
G(A x, B y, C z) \leq & \alpha\{G(x, y, z)+G(x, B y, C z)+G(x, A x, A x)\}+ \\
& \beta\{\max \{G(y, B y, C z), G(B y, y, z), G(y, A x, A x), G(C z, x, z)\}\}+ \\
& \gamma\{G(x, B y, y)++G(y, C z, z)+G(z, A x, A x)\}
\end{aligned}
$$

$\forall x, y, z \in X$, where $\alpha, \beta, \gamma>0$ and $6 \alpha+4 \beta+5 \gamma<\frac{1}{2}$. Then mappings $A, B$ and $C$ have a unique common fixed point in $X$.

Remark 3.1. Theorem(3.1) and Corollary(3.1) improve and generalize the corresponding results of Abbas and Rhoades[6], Mustafa et al.[13], Chugh, Kadian, Rani and Rhoades[7].

Theorem 3.2. Let $(X, G)$ be a complete $G$-metric space and let $A, B, C, R$, $S$ and $T$ be six mappings on $X$ into itself satisfying the following conditions:

(i) $A(X) \subseteq T(X), B(X) \subseteq S(X), C(X) \subseteq R(X)$.

(ii) The pairs $(A, R),(B, T)$ and $(C, S)$ are commuting mapping. 
(iii) $\forall x, y, x \in X$

$$
\begin{aligned}
G\left(A^{m} x, B^{m} y, C^{m} z\right) \leq & \alpha\left\{G(R x, T y, S z)+G\left(R x, B^{m} y, C^{m} z\right)\right. \\
& \left.+G\left(R x, A^{m} x, A^{m} x\right)\right\}+ \\
& \beta\left\{\operatorname { m a x } \left\{G\left(T y, B^{m} y, C^{m} z\right), G\left(B^{m} y, T y, S z\right),\right.\right. \\
& G\left(T y, A^{m} x, A^{m} x\right), \\
& \left.\left.G\left(C^{m} z, R x, S z\right)\right\}\right\}+\gamma\left\{G\left(R x, B^{m} y, T y\right)+G\left(T y, C^{m} z, S z\right)\right. \\
& \left.+G\left(S z, A^{m} x, A^{m} x\right)\right\}
\end{aligned}
$$

where $\alpha, \beta, \gamma>0$ and $6 \alpha+4 \beta+5 \gamma<\frac{1}{2}, m \in N$ then $A, B, C, R, S$ and $T$ have a unique fixed point in $X$.

Proof. Suppose the mappings $A, B, C, R, S$ and $T$ satisfy inequality(3.7).

Since $A^{m}(X) \subset A^{m-1}(X) \subset A^{m-2}(X) \subset \ldots \subset A(X) \subseteq T(X)$. Similarily we can show that $B^{m}(X) \subset S(X)$ and $C^{m}(X) \subset R(X)$. From Theorem(3.1), we can show that $A^{m}, B^{m}, C^{m}, R, S$ and $T$ have a unique fixed point $u$ in $X$.

Now using inequality (3.7), we have

$$
\begin{aligned}
& G\left(A^{m} A u, B^{m} u, C^{m} u\right) \leq \alpha\left\{G(R A u, T u, S u)+G\left(R A u, B^{m} u, C^{m} u\right)\right. \\
& \left.+G\left(R A u, A^{m} A u, A^{m} A u\right)\right\}+ \\
& \beta\left\{\operatorname { m a x } \left\{G\left(T u, B^{m} u, C^{m} u\right), G\left(B^{m} u, T u, S u\right), G\left(T u, A^{m} A u, A^{m} A u\right),\right.\right. \\
& \left.\left.G\left(C^{m} u, R A u, S u\right)\right\}\right\}+\gamma\left\{G\left(R A u, B^{m} u, T u\right)+G\left(T u, C^{m} u, S u\right)+\right. \\
& \left.G\left(S u, A^{m} A u, A^{m} A u\right)\right\}
\end{aligned}
$$

By condition(ii) we have $R A u=A R u=A u$ and $A u=A\left(A^{m} u\right)=A^{m+1} u=$ $A^{m} A u$ then we get

$$
\begin{aligned}
G(A u, u, u) & \leq \alpha\{G(A u, u, u)+G(A u, u, u)+G(A u, A u, A u)\}+\beta\{\max \{G(u, u, u) \\
& G(u, u, u), G(u, A u, A u), G(u, A u, u)\}\}+\gamma\{G(A u, u, u)+ \\
& G(u, u, u)+G(u, A u, A u)\} \\
& \leq 2 \alpha G(A u, u, u)+2 \beta G(A u, u, u)+3 \gamma G(A u, u, u) \\
& \leq(2 \alpha+2 \beta+3 \gamma) G(A u, u, u)
\end{aligned}
$$

This implies that $G(A u, u, u)=0, \Rightarrow A u=u$.

Similarily we can show that $u=B u$ and $u=C u$.

Thus we have $u=A u=B u=C u=R u=S u=T u$. So that $A, B, C, R, S$ and $T$ have a common fixed point in $X$. 
Let $v$ be another common fixed point of $A, B, C, R, S$ and $T$ then using the inequality(3.6), we have

$$
\begin{aligned}
G(v, u, u) & \leq \alpha\left\{G(R v, T u, S u)+G\left(R v, B^{m} u, C^{m} u\right)+G\left(R v, A^{m} v, A^{m} v\right)\right\}+ \\
& \beta\left\{\operatorname { m a x } \left\{G\left(T u, B^{m} u, C^{m} u\right), G\left(B^{m} u, T u, S u\right), G\left(T u, A^{m} v, A^{m} v\right),\right.\right. \\
& \left.\left.G\left(C^{m} u, R v, S u\right)\right\}\right\}+\gamma\left\{G\left(R v, B^{m} u, T u\right)+G\left(T u, C^{m} u, S u\right)\right. \\
& \left.+G\left(S u, A^{m} v, A^{m} v\right)\right\} \\
& \leq \alpha\{G(v, u, u)+G(v, u, u)+G(v, v, v)\} \\
& +\beta\{\max \{G(u, u, u), G(u, u, u), \\
& G(u, v, v), G(u, v, u)\}\}+\gamma\{G(v, u, u)+G(u, u, u)+G(u, v, v)\} \\
& \leq(2 \alpha+2 \beta+3 \gamma) G(v, u, u)
\end{aligned}
$$

This implies that $G(v, u, u)=0 \Rightarrow v=u$.

Thus $u$ is the unique common fixed point of $A, B, C, R, S$ and $T$.

Corollary 3.2. Let $(X, G)$ be a complete $G$-metric space and let $A$ be a mapping of $X$ into itself satisfying the following conditions

$$
\begin{aligned}
G\left(A^{m} x, A^{m} y, A^{m} z\right) \leq & \alpha\left\{G(x, y, z)+G\left(x, A^{m} y, A^{m} z\right)+G\left(x, A^{m} x, A^{m} x\right)\right\}+ \\
& \beta\left\{\operatorname { m a x } \left\{G\left(y, A^{m} y, A^{m} z\right), G\left(A^{m} y, y, z\right), G\left(y, A^{m} x, A^{m} x\right),\right.\right. \\
& \left.\left.G\left(A^{m} z, x, z\right)\right\}\right\}+\gamma\left\{G\left(x, A^{m} y, y\right)+G\left(y, A^{m} z, z\right)\right. \\
& \left.+G\left(S z, A^{m} x, A^{m} x\right)\right\}
\end{aligned}
$$

$\forall x, y, z \in X$, where $\alpha, \beta, \gamma>0$ and $6 \alpha+4 \beta+5 \gamma<\frac{1}{2}, m \in N$ then $A$ has a unique fixed point in $X$.

\section{References}

[1] B.C. Dhage, Generalized metric spaces and mappings with fixed point, Bull. Calcutta Math. Soc., 84, No.4,(1992), 329-336.

[2] B.C. Dhage, On generalized metric spaces and topological structure II. Pure. Appl. Math. Sci. 40,No.1-2(1994),37.

[3] B.C. Dhage, A common fixed point principle in D-metric spaces, Bull.Cal. Math.Soc., 91,No.6(1999),475-480.

[4] B.C. Dhage, Generalized metric spaces and topological structure I, $A n$ nalele Stiintifice ale Universitatii Al.I. Cuza, 46,No.1(2000),3-24. 
[5] M. Abbas, T. Nazir, P. Vetro, Common fixed point results for three maps in G-metric spaces. Filomat, 25, No.4(2011), 1-17

[6] M. Abbas, B.E. Rhoades, Common fixed point results for noncommuting mappings without continuity in generalizes metric spaces, Appl. Math. Comput., 215,(2009),262-269

[7] R. Chugh, T. Kadian, A. Rani and B.E. Rhoades, Property in G- metric spaces, Fixed point theory and Applications, Article ID 401684,(2010), 12 Pages.

[8] S.V.R. Naidu, K.P.R. Rao and N. Srinivasa Rao, On the topology of Dmetric spaces and the generation of D-metric spaces from metric spaces , Internat. J. Math. Math. Sci., No. 51(2004), 2719.

[9] S.V.R. Naidu, K.P.R. Rao and N. Srinivasa Rao, On the concepts of balls in a D-metric space, Internat. J. Math. Math. Sci.,2005,No.1(2005), 133.

[10] S.V.R. Naidu, K.P.R. Rao and N. Srinivasa Rao, On convergent sequences and Fixed point theorems in D-metric spaces, Internat. J. Math. Math. Sci.,2005,12(2005), 1969.

[11] Wasif Shatanawi, Fixed point theory for contractive mappings satisfying $\phi$ - maps in G-metric spaces, fixed point theory and Applications, Article ID 181650,(2010), 9 Pages.

[12] Zaed Mustafa and Brailey Sims, A new approach to generalized metric spaces, Journal of Nonlinear and Convex Analysis, 7,No.2(2006),289-297.

[13] Zaed Mustafa, Hamed Obiedat and Fadi Awawdeh, Some fixed point theorem for mapping on complete G-metric spaces, Fixed point theory and Applications,Article ID 189870,(2008),12 Pages.

[14] Zaed Mustafa, Wasfi Shatanawi and Bataineh Malik,. Existence of fixed point results in G-metric spaces, Internat. J. Math. Sci., Article ID 283028,(2009),10 Pages.

[15] Zaed Mustafa and Brailey Sims, Fixed point theorems for contractive mappings in complete G-metric spaces, Fixed point theory and Applications, Article ID 917175,(2009), 10 Pages. 